\title{
An empirical examination of the management of return of individual research results and incidental findings in genomic biobanks
}

\author{
Gina Johnson, $\mathrm{PhD}^{1}$, Frances Lawrenz, $\mathrm{PhD}^{1}$ and Mao Thao, BA, BS ${ }^{1}$
}

Purpose: The purpose of this study was to examine and document the management and return of individual research results and incidental findings to research participants among biobanks.

Methods: A comprehensive Internet search was completed to identify biobanks and collect available documents about biobanks and their procedures and policies regarding the return of results. The Internet search was supplemented by an e-mail request to gather further such documents. A total of 2,366 documents were collected for analysis from a sample of 85 biobanks.

Results: The major finding of this empirical work is that the majority of the biobanks in the sample do not address the return of individual research results and incidental findings in their publicly available documents.

Conclusion: The results suggest that there is a need for more discussion and guidance about the most appropriate ways for biobanks to consider the return of individual research results and incidental findings and generate policies and procedures that address this issue.

Genet Med 2012:14(4):444-450

Key Words: biobank; genomic research; incidental findings; individual research results

\section{INTRODUCTION}

Research with human biological materials has been important in identifying genetic relationships with diseases or susceptibility to diseases ${ }^{1}$ and in developing new approaches to therapy such as pharmacogenetics. ${ }^{2,3}$ Scientists are increasingly assembling large biobanks and archiving DNA and health information. ${ }^{1}$ Genetic research with stored biological materials raises ethical concerns. For example, the National Marrow Donor Program re-contacted thousands of donors because samples had been collected without consent. ${ }^{4}$ In Canada, the First Nations people demanded return of their stored biological materials after learning that researchers who had collected the materials to study rheumatoid arthritis had also used them in other research. ${ }^{5}$

Researchers struggle with issues of returning individual research results (IRRs) and incidental findings (IFs) to research participants. In the past, IFs were largely ignored and the duty to return IRRs was often expressly disclaimed by investigators. ${ }^{6}$ The literature distinguishes return of aggregate results from return of individual results. ${ }^{7-9}$ Some research indicates that participants appear to want more than aggregated research results. ${ }^{10-13}$ However, returning individual results may produce anxiety and involve cost, burden, and challenges to investigators. There are debates about requiring that a lab be Clinical Laboratory Improvement Amendments-approved in order to return genetic results. ${ }^{6,14,15}$ Richardson and Belsky have argued that researchers owe some clinical information to participants. ${ }^{16}$
They, along with a number of other commentators, maintain that the individuals who could most directly benefit from the information should have access to it. ${ }^{16-20}$ Others argue that returning results might cause more harm than good if the results are not carefully validated. ${ }^{21}$ Further debate addresses the appropriateness of stripping samples of identifiers, whether anonymization is appropriate for archived samples, what the informed consent process should say about IFs and IRRs and the duties of institutional review boards, biobanks, and investigators. ${ }^{22}$

The literature reveals widespread concern over the lack of guidance on these issues. ${ }^{23-25}$ Yet to date, few studies have empirically examined how these ethical issues are being addressed, particularly with regard to genetic research with biological materials stored in biobanks. Focused examination of the publically available policies of biobanks is essential. Policies that are not publicly available cannot form a basis for collective discussion and consideration in the debate.

This study was designed to provide empirical evidence on the publicly available policies of biobanks in order to provide information for models, guidance, and recommendations on next steps for biobanks. It builds on prior work ${ }^{6}$ about the return of IFs and IRRs and was guided by a working group of genetic and ethics professionals as part of a National Institutes of Health (NIH) grant (no. 2-R01-HG003178). Because of the focus on publicly available documentation, Web-based searching supplemented by e-mail requests for other publicly available 
documents was determined to be the most appropriate methodology to obtain information. Although survey or interview methodology might have produced more in-depth information, that information would not necessarily be what would be publicly available. A detailed coding form was used to document the content of the publicly available documents posted at biobank websites (see Supplementary Data online). The content was coded to address biobank policy on the management of IRRs and IFs in research and in reanalysis by secondary researchers after initial collection of the samples and data. For the purposes of this study, a biobank was defined as a project that collects or archives human DNA samples or data for the purpose of genetic research. To qualify as a biobank for the purposes of this analysis, the biobank had to hold at least 500 samples or include at least 500 participants. An IF was defined as “a finding concerning an individual research participant [or here, an individual contributor] that has potential health or reproductive importance and is discovered in the course of conducting research but is beyond the aims of the study." ${ }^{\circ} \mathrm{An}$ IRR was "a finding concerning an individual contributor that has potential health or reproductive importance and is discovered in the course of research on the focal variables under study in meeting the stated aims of the research project." 26 The primary research questions for this study were as follows:

- How do documents publicly available from biobanks through Internet searches and e-mail query describe biobank policies on the return of IRRs and IFs among biobanks in the United States and beyond?

- How do documents publicly available from biobanks address IRRs and IFs in the reanalysis of data and samples by secondary researchers?

\section{MATERIALS AND METHODS}

From November 2009 to July 2010, a comprehensive Internet search was completed using Google and the website tools of the NIH websites (including the websites of the NIH's institutes). Eighteen separate terms were used to create a list of biobanks from three different groups: NIH intramural (43 biobanks); other US biobanks, i.e., those not coordinated intramurally through NIH (66 biobanks); and non-US biobanks (12 biobanks). The search terms used were biobank, biorepository, consortium, DNA bank, DNA databank, DNA database, gene bank, gene databank, gene database, genetic bank, genetic databank, genetic database, genomic bank, genomic databank, genomic database, registry, repository, and tissue bank. Results are presented in the following for each group separately. The other US biobank and non-US biobank groups were broadly defined and included notfor-profit, publicly funded, and direct-to-consumer and other for-profit entities as all types of biobanks were the target of this research. During the search, the first 100 links provided by Google for each of the 18 separate terms used in the search process were followed and, when a link led to a biobank, this biobank was included in the master list (see Supplementary Data online).
From April to September 2010, biobanks were categorized based on the information included on their websites. Categories are presented in Table 1 and include such things as age of biobank and amount and type of samples. A total of 2,366 documents were collected from the websites of each of the 43 $\mathrm{NIH}$ intramural and 12 non-US biobanks, as well as from a random sample of 30 of the other US biobanks for a total of 85 biobanks. After the list of 66 other (non-NIH) US biobanks was generated from the Internet search, a random-number function in Microsoft Excel was used to select 30 of these biobanks from which to collect documents. A comparison was made between the random sample of 30 other US biobanks and the 36 biobanks that were not sampled to determine whether the sampled biobanks represented the group as a whole; that analysis showed the random sample of 30 was representative.

The documents collected included Web pages and linked documents (e.g., Adobe pdfs, Microsoft Word documents). The documents were coded using the NVivo software (QSR International, Cambridge, MA). From September to October 2010, e-mail solicitations were sent to all of the biobanks, requesting additional publicly available documents not posted on the biobank's website. The text of the e-mail requested additional documents related to return of research results or IFs that could include, for example, policies, consent forms, consent form templates, research and informed consent protocols, or material transfer agreements. The request was sent to provide the biobanks the opportunity to share their most up-to-date publicly available documents in the event that they were not posted to the Internet. Forty-six biobanks responded to the e-mail request and sent a total of 29 additional documents, for a response rate of $54 \%$. Documents received were coded in the same manner as the documents from the websites. In coding all documents, whether obtained from the Internet or received by e-mail, query rater reliability checks showed an intrarater reliability of 0.97 and inter-rater reliability of 0.94 . It should be noted that the quotations used in the results section are from the documents collected in 2010 and may have been from works in progress or model language and may not be reflective of currently posted wordings.

\section{RESULTS}

Overall, the majority of the biobanks in the sample did not address issues related to the return of IRRs and IFs. Many of the coding questions were not addressed by most of the biobanks in the study. Those that did address the return of IRRs and IFs mostly indicated that they did not return either to the biobank participants.

Table 1 provides a cross-comparison of specific biobank characteristics. Among these, $51 \%$ were $<10$ years old. Almost half of the biobanks (45\%) conducted general genetic health studies rather than studying a specific disease, although $72 \%$ of the NIH intramural biobanks studied a disease or group of diseases. Slightly more than half (56\%) of all the biobanks had sample sizes $>10,000$ and $91 \%$ of them contained samples with or without accompanying data, rather than containing only genetic data. Half of all the biobanks (50\%) collected 
Table 1 Comparison of characteristics by type of biobank

\begin{tabular}{|c|c|c|c|c|c|c|c|c|}
\hline \multirow{3}{*}{ Age of biobank } & \multicolumn{2}{|c|}{ NIH } & \multicolumn{2}{|c|}{ Other US } & \multicolumn{2}{|c|}{ Non-US } & \multicolumn{2}{|c|}{ Total } \\
\hline & $n$ & $\%$ & $n$ & $\%$ & $n$ & $\%$ & $n$ & $\%$ \\
\hline & $N=19$ & & $N=11$ & & $N=9$ & & $N=39$ & \\
\hline $5-9$ years & 3 & 16 & 2 & 18 & 2 & 22 & 7 & 18 \\
\hline 10 or more years & 9 & 47 & 5 & 45 & 5 & 56 & 19 & 49 \\
\hline Single disease/disorder & 16 & 37 & 6 & 20 & 0 & 0 & 22 & 26 \\
\hline Class of diseases/disorders & 15 & 35 & 8 & 27 & 2 & 17 & 25 & 29 \\
\hline General genetic studies & 12 & 28 & 16 & 53 & 10 & 83 & 38 & 45 \\
\hline Sample size ${ }^{a}$ & $N=25$ & & $N=16$ & & $N=7$ & & $N=48$ & \\
\hline$>100,000$ & 2 & 8 & 4 & 25 & 4 & 57 & 10 & 21 \\
\hline Biobank contents & $N=41$ & & $N=29$ & & $N=12$ & & $N=82$ & \\
\hline Genomic data only & 7 & 17 & 0 & 0 & 0 & 0 & 7 & 9 \\
\hline $\begin{array}{l}\text { Biological samples } \\
\text { (with or without data) }\end{array}$ & 34 & 83 & 29 & 100 & 12 & 100 & 75 & 91 \\
\hline Age of participants & $N=19$ & & $N=17$ & & $N=6$ & & $N=42$ & \\
\hline Adults only & 6 & 32 & 10 & 59 & 5 & 83 & 21 & 50 \\
\hline Adults and children & 13 & 68 & 7 & 41 & 1 & 17 & 21 & 50 \\
\hline
\end{tabular}

This information was gathered from the biobank websites, not from subsequent e-mail contact. $N$ represents the number of biobanks that had information on their websites. Some percentage totals do not add to $100 \%$ due to rounding. NIH, National Institutes of Health.

aBiobanks needed to have at least 500 samples or participants to be included in this study.

samples/data only from adults. Almost two-thirds of all the biobanks $(63 \%)$ consented participants themselves rather than creating a biobank from existing samples/data collected by outside researchers.

\section{Return of information to participants}

Table 2 reflects the number of biobanks in each group that had statements regarding the return of some type of information to participants followed by numbers of biobanks having statements about returning specific types of information. Statements about the return of specific types of information are not mutually exclusive so the numbers do not necessarily total to the numbers returning some type of information. The coding was very strict. A statement had to explicitly mention "individual research results" or "individual incidental findings" to be coded as IRR or IF. If the statement just said "results," it was coded as possibly referring to IRR, IF, or aggregated results, in other words, as unclear. Statements about return of information from reanalysis are analyzed similarly in the section "Return of information to participants in data/sample reanalysis". Some quotations are included for illustrative purposes.

NIH intramural biobanks. Of the 43 NIH intramural biobanks, 21 or $49 \%(21 / 43)$ mentioned whether or not they would return information of some sort to participants.

Yes: Of these 21, 7 indicated that they would return information to participants. The documents from these seven were also coded for more specific circumstances. Four of these seven stated that they would return results of screening required for participation in the study. No biobank stated specifically that it would return IRRs. One, GENEVA (a network of biobanks), stated that it would return IFs to participants when possible. Four of the seven indicated that they would return information of some sort to participants in some circumstances, but whether this information referred to IRRs, IFs, or both was not clear. The National Children's Study stated "if a test, measurement, 
Table 2 Return of information to participants by type of biobank

\begin{tabular}{|c|c|c|c|c|c|c|c|c|c|}
\hline & \multicolumn{3}{|c|}{$\begin{array}{c}\text { NIH }(n=43)^{\text {a }} ; \\
\text { ratio (percentage) }\end{array}$} & \multicolumn{3}{|c|}{$\begin{array}{l}\text { Other US }(n=30) \text { a } \\
\text { ratio (percentage) }\end{array}$} & \multicolumn{3}{|c|}{$\begin{array}{l}\text { Non-US }(n=12) \text { a; } \\
\text { ratio (percentage) }\end{array}$} \\
\hline & Yes $^{b}$ & $\mathrm{No}^{b}$ & NA & Yes $^{b}$ & $\mathrm{No}^{\mathrm{b}}$ & NA & Yes $^{b}$ & $\mathrm{No}^{\mathrm{b}}$ & NA \\
\hline
\end{tabular}

If return of information is mentioned

\begin{tabular}{|c|c|c|c|c|c|c|c|c|c|}
\hline Will screening results be returned? & 4/21 (19) & $0 / 21(0)$ & - & $3 / 13(23)$ & $0 / 13(0)$ & - & 2/6 (33) & $0 / 6(0)$ & - \\
\hline Will IRRs be returned? & $0 / 21(0)$ & $11 / 21(52)$ & - & $2 / 13(15)$ & $5 / 13(38)$ & - & $1 / 6(17)$ & $3 / 6(50)$ & - \\
\hline Will IFs be returned? & $1 / 21(5)$ & $4 / 21(19)$ & - & $2 / 13(15)$ & $0 / 13(0)$ & - & $0 / 6(0)$ & $0 / 6(0)$ & - \\
\hline $\begin{array}{l}\text { Will information that is not clearly } \\
\text { defined as either an IRR or IF be } \\
\text { returned? }\end{array}$ & 4/21 (19) & $3 / 21(14)$ & - & $2 / 13(15)$ & $2 / 13(15)$ & - & $1 / 6(17)$ & $1 / 6(17)$ & - \\
\hline
\end{tabular}

IF, incidental finding; IRR, individual research result; NA, not applicable; NIH, National Institutes of Health.

${ }^{a}$ The sample size $(n)$ represents the number of biobanks from which documents were obtained. ${ }^{b}$ The yes and no options for the questions specific to IRRs, IFs, and information not clearly an IRR or an IF do not add to the total number of biobanks in the first row because some biobanks had statements in multiple categories or none.

Two of the six biobanks that addressed the return of information said both yes and no.

or collection is performed, and the results indicate a known health effect or risk to the participant that is clinically relevant and actionable, the Study is obligated to reveal the finding to the participant." The remaining three biobanks with unclear statements indicated that they would return information to an individual if the situation warranted it. These biobanks included the eMERGE Network, the Framingham Heart Study, and the Cancer Human Biobank, which also indicated that the intent was not to return IRRs but that there may be occasions when findings would need to be shared with participants.

No: Of the $21 \mathrm{NIH}$ intramural biobanks that mentioned whether or not they would return information to participants, 14 stated that they would not return information. Of the 14, 11 indicated that they would not return IRRs to participants. Four indicated that they would not return IFs to individual participants. Three indicated that they would not return information to participants, but it was not clear what type of information they were referring to.

Other US biobanks. Of the 30 other US biobanks, 13 or $43 \%(13 / 30)$ mentioned whether or not they would return information to participants.

Yes: Of these 13, 6 indicated that they would return information to participants. Three stated that they would return screening results required for participation. Two stated specifically that they would return IRRs, including the Personalized Medicine Research Project at Marshfield Clinic and 23andMe. Marshfield Clinic returns IRRs only to participants whose results warrant return for medical reasons. Two biobanks, the Personalized Medicine Research Project at Marshfield Clinic and the University of Connecticut Behavioral Gene Bank, specifically stated that IFs would be returned. Two biobanks, SFARI Base and the Kaiser Permanente Research Program on Genes, Environment, and Health, indicated that they would return some sort of information to participants, at least in some circumstances.
The Kaiser Permanente Research Program on Genes, Environment, and Health indicated that IRRs would not be generally returned to all participants, "however, if scientists discover information as a result of RPGEH research that we believe is of substantial medical importance to you, we may contact you and ask if you want to learn the results."

No: Of the 13 other US biobanks that mentioned whether they would return information of some sort or not to participants, 7 stated that they would not return information. Five biobanks specifically indicated that they would not return IRRs to participants. No biobank indicated specifically that it would not return IFs to participants. Two biobanks, Asterand and the National Psoriasis Victor Henschel BioBank, indicated that they would not return information to participants. Asterand stated, "neither you nor your study doctor will be given information obtained from the research conducted with your samples and data." The National Psoriasis Victor Henschel BioBank stated, "You will not receive any information from your donated samples. You will not receive results on the research performed using your samples."

Non-US biobanks. Of the 12 non-US biobanks, 6 or $50 \%(6 / 12)$ mentioned whether or not they would return information of some sort to participants. Of these six biobanks, two had policies that produced both yes and no responses on the more specific coding items.

Yes: Of these six, four indicated that they would return information to participants. Two stated that they would return results of screening required for participation. One biobank, the Estonian Genome Project, specifically mentioned that it would return IRRs if requested by participants, stating, "gene donors have the right to access personally their data stored in the Gene Bank." No non-US biobanks specifically stated that they would return IFs to participants. The Latvian Genome Project indicated that it would return 
some sort of information to participants, at least in some circumstances.

No: Of the six non-US biobanks that mentioned whether or not they would return information to participants, four stated that they would not return this information. As mentioned earlier, two of the six non-US biobanks stated that they would return one type of information but would not return another, so there is overlap. Three of the biobanks specifically stated that IRRs would not be returned. None of the biobanks specifically stated that they would not return IFs. One biobank, the Western Australia DNA Bank, indicated that it would not return information to participants. Its documents stated, "the WA DNA Bank itself does not provide any information or results that have arisen from research carried out on the DNA stored within the facility."

\section{Return of information to participants in data/sample reanalysis}

Table 3 reflects the number of biobanks in each group that had statements regarding return of information to participants that specifically resulted from reanalysis of the data and/or samples by secondary researchers. Due to the archival nature of some biobanks, it was sometimes difficult to ascertain which statements addressed future reanalysis of the data/samples. As a result, only those statements that clearly referred to future reanalysis using terms such as "future studies," "secondary analysis," "reexamination," and "other researchers" were coded as reanalysis.

$\mathrm{NIH}$ intramural biobanks. Of the $43 \mathrm{NIH}$ intramural biobanks, 6 or 14\% (6/43) mentioned whether or not they would return information of some sort to participants related to the reanalysis of data and/or samples.

Yes: Of these six, five indicated that they would return information to participants. GENEVA specifically indicated that it would consider returning IFs from reanalysis.

The Prostate SPORE NBN Pilot indicated that it would return both IRRs and IFs. The documents collected from the
National Children's Study and the Cancer Human Biobank also indicated they would return information from reanalysis. The Cancer Human Biobank indicated that if results from a secondary investigator warranted returning these results to the participant, the biobank would do so.

No: One NIH Intramural biobank, the National Institute of Diabetes and Digestive and Kidney Diseases Central Repository, indicated that it would not return information obtained in reanalysis. Documents stated, "because other researchers will not have access to your identity, neither you nor your physician will get the eventual results of studies that might be performed using your sample."

Other US biobanks. None of the 30 other US biobanks, $0 \%$ $(0 / 30)$, addressed return of any information from reanalysis of biobank data/samples.

Non-US biobanks. Of the 12 biobanks in the non-US biobank group, 1 or $8 \%(1 / 12)$ addressed return of information from reanalysis of biobank data/samples. In its documents, CARTaGENE stated, "no results from future research projects using data or samples will be communicated to participants by CARTaGENE."

\section{DISCUSSION}

The most important findings from our research are that there are few differences among the three different groups of biobanks (NIH intramural, other US, and non-US), only about half of the biobanks address the return of IFs or IRRs in their publicly available documents, and few biobanks suggest that IFs or IRRs should be returned.

The comparison across the three groups-NIH intramural biobanks, other US biobanks, and non-US biobanks-was conducted because these groups represent different portions of the population of biobanks and different search techniques, and their policies might differ accordingly. In addition, other published work ${ }^{27,28}$ had suggested that non-US biobanks might have different perspectives. The data presented earlier, however,

Table 3 Return of reanalysis information to participants by type of biobank

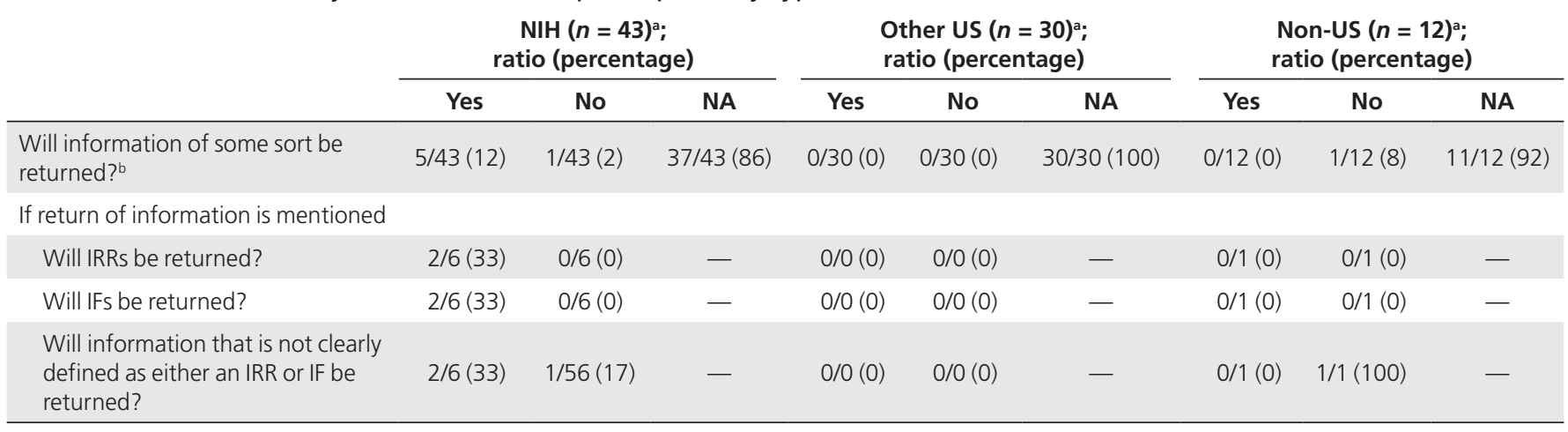

IF, incidental finding; IRR, individual research result; NA, not applicable; $\mathrm{NIH}$, National Institutes of Health.

aThe sample size $(n)$ represents the number of biobanks from which documents were obtained. ${ }^{\circ}$ The yes and no options for the questions specific to IRR, IF, and unclear language do not add to the total number of biobanks in the first row because some biobanks had statements in multiple categories or none. 
show only few and minor differences among the groups. This suggests that the biobanks had similar policies about IRRs and IFs. However, few of the issues were addressed at all in the documents from any group, so it is impossible to support this conclusion. The striking similarity across all three groups was their lack of publicly available policies on IFs and IRRs.

In conducting this study, we found that biobanks are split fairly evenly on whether or not they even address return of IFs and IRRs. Fewer address the return of IFs and IRRs in future analyses. This lack of information suggests several possibilities. Biobanks may not believe this information should be made publicly available, they may have not determined how to address the return of IFs and IRRs, or they may not believe they need to address the return of IFs and IRRs. Whatever the case, it seems that more discussion and awareness about the importance of biobanks having policies on IFs and IRRs is critical for the future. The need may be even more acute for discussion about the importance of policy about the return of IFs and IRRs in reanalysis. Among those biobanks that do address return of information about IFs and IRRs, some return no findings at all whereas others return some types of information. The majority of the biobank policies suggest that IRRs will not be returned. Certainly contacting research subjects who may have provided their materials to a researcher other than the biobank or provided them in the long past can be difficult. In addition, the decision to return or not is an important ethical issue that deserves careful consideration. It is clear from our findings that there is not consensus yet about what biobank policy should say about the return of IFs and IRRs. This further supports the idea that more discussion and awareness about the importance of developing sound policy on IFs and IRRs is necessary.

In coding the documents it was often difficult to distinguish between IRRs and IFs. This is understandable because it can be difficult to identify what is "beyond the aims of the study," especially when the entire genome is under scrutiny and the research is not hypothesis driven but more exploratory or inductive. ${ }^{6,29,30}$ Moreover, recruitment for biobank participation may be for an open-ended array of future studies. This distinction between IRRs and IFs may not be necessary. It is also possible that different types of distinctions might be more fruitful. Research subjects may want individual results that have clinical meaning for their health but may not care whether those findings are results that are generated as a result of the primary aims of the study or are IFs of clinical significance.

The lack of specificity in the publicly available documents may link into the debate about whether or not to return any type of results and ultimately to whether biobank data should be identifiable at all. Some commentators suggest that researchers may be obligated to return at least some results. ${ }^{16}$ Others argue that before any results could be returned they would have to be carefully validated because otherwise harms may accrue. Another component of the debate is about maintaining identifiers and how this relates to the trade-off between privacy and confidentiality and any perceived benefit from returning individual results that may be generated. ${ }^{31}$ The biobanks are in the middle of this debate and have consequently developed mixed approaches to dealing with the return of results.

This study was an effort to search for and create a representative list of biobanks with websites from three major categories of biobanks and to gather their publicly available policies to determine how they approach the return of IFs and IRRs. The overarching finding, despite the large number of biobank documents analyzed, is that half of the biobanks in the sample did not address the return of IRRs and IFs in their publicly available documents, at least as of 2010. This finding, along with the newly emerging issues and complexities of returning information to participants, indicates that how biobanks should approach the return of IFs and IRRs requires further discussion, and ultimately guidance. Given the growing agreement that researchers have a responsibility to determine and make clear to research participants whether or not IFs and IRRs will be returned, ${ }^{32-36}$ it seems reasonable to suggest that biobanks should be more explicit about their policies in this regard.

\section{Limitations}

There are several methodological issues that constrain this study. Google utilizes the user to prioritize results, thereby customizing searches to some extent, and also moves results to the top of the list if the user has previously followed the links. These prioritization-of-result issues were somewhat resolved by using the same computer to complete all the searches, as well as completing each search and following all 100 links presented in one sitting. Another limitation is that the websites that were identified and the documents returned in response to the e-mail request may only partially reflect actual policy. For example, biobanks that aggregate archival materials previously collected during routine care may not post policy information to their websites. In addition, the biobanks sampled were limited to only those containing 500 or more samples or participants.

The limited time frame of the study, from November 2009 to October 2010, is also a constraint because the debate about IFs/IRRs in biobanks is ongoing and policies are probably evolving. The language we quote was what we found in 2010; some of those documents may have been works in progress, model statements, or since superceded. Finally, the non-US results are limited to English-language websites and documents.

\section{SUPPLEMENTARY MATERIAL}

Supplementary material is linked to the online version of the paper at http://www.nature.com/gim

\section{ACKNOWLEDGMENTS}

Deepest appreciation is extended to Susan Wolf, Jeff Kahn, Brian Van Ness, Pari McGarraugh, and Leili Fatehi for their support and assistance. Preparation of this article was supported by National Institutes of Health (NIH)/National Human Genome Research 
Institute (NHGRI) grant no. 2-R01-HG003178-03 on "Managing Incidental Findings and Research Results in Genomic Biobanks \& Archives" (S. Wolf, principal investigator).

The views expressed in this article are those of the authors and not necessarily those of the $\mathrm{NIH}$ or the NHGRI.

\section{DISCLOSURE}

The authors declare no conflict of interest.

\section{REFERENCES}

1. Wolf LE, Lo B. Untapped potential: IRB guidance for the ethical research use of stored biological materials. IRB 2004;26:1-8.

2. Wolf $C R$, Smith $G$, Smith RL. Science, medicine, and the future: pharmacogenetics. BMJ 2000;320:987-990.

3. Meyer UA. Pharmacogenetics and adverse drug reactions. Lancet 2000;356:1667-1671.

4. Otto MA. Informed consent: failure to obtain consent derails bone marrow research repository. Med Res L Pol'y Rep (BNA) 2003;2:206.

5. Dalton R. Tribe blasts 'exploitation' of blood samples. Nature 2002;420:111

6. Wolf SM, Lawrenz FP, Nelson CA, et al. Managing incidental findings in human subjects research: analysis and recommendations. J Law Med Ethics 2008;36:216-248.

7. Fernandez CV, Kodish E, Weijer C. Informing study participants of research results: an ethical imperative. IRB 2003;25:12-19.

8. Eiseman E, Broom G, Brower J, et al. Case Studies of Existing Human Tissue Repositories: "Best Practices" for a Biospecimen Resource for the Genomic and Proteomic Era. RAND: Santa Monica, CA, 2003.

9. Haga SB, Beskow LM. Ethical, legal, and social implications of biobanks for genetics research. Adv Genet 2008;60:505-544.

10. Shalowitz DI, Miller FG. Communicating the results of clinical research to participants: attitudes, practices, and future directions. PLoS Med 2008;5:e91.

11. Kaufman D, Murphy J, Scott J, Hudson K. Subjects matter: a survey of public opinions about a large genetic cohort study. Genet Med 2008;10: 831-839.

12. Murphy J, Scott J, Kaufman D, Geller G, LeRoy L, Hudson K. Public expectations for return of results from large-cohort genetic research. Am J Bioeth 2008;8:36-43.

13. Wendler D, Emanuel E. The debate over research on stored biological samples: what do sources think? Arch Intern Med 2002;162: 1457-1462.

14. Clayton EW. Incidental findings in genetics research using archived DNA. J Law Med Ethics 2008:36:286-291, 212.

15. Clinical Laboratory Improvement Amendments (CLIA) of 1988. 42 U.S. Code 263a. 2008.

16. Richardson HS, Belsky L. The ancillary-care responsibilities of medical researchers. An ethical framework for thinking about the clinical care that researchers owe their subjects. Hastings Cent Rep 2004;34:25-33.
17. Church GM. The personal genome project. Mol Syst Biol 2005; $1: 2005.0030$

18. Church GM. Genomes for all. Sci Am 2006:294:46-54

19. Fabsitz RR, McGuire A, Sharp RR, et al. Ethical and practical guidelines for reporting genetic research results to study participants: updated guidelines from a National Heart, Lung, and Blood Institute working group. Circ Cardiovasc Genet 2010;3:574-580.

20. Kohane IS, Mandl KD, Taylor PL, Holm IA, Nigrin DJ, Kunkel LM. Medicine. Reestablishing the researcher-patient compact. Science 2007:316:836-837.

21. Clayton EW, Ross LF. Implications of disclosing individual results of clinical research. JAMA 2006;295:37; author reply 37-38.

22. Wolf LE, Bouley TA, McCulloch CE. Genetic research with stored biological materials: ethics and practice. IRB 2010;32:7-18

23. Andrews LB. Harnessing the benefits of biobanks. J Law Med Ethics 2005:33:22-30.

24. Cambon-Thomsen A, Rial-Sebbag E, Knoppers BM. Trends in ethical and legal frameworks for the use of human biobanks. Eur Respir J 2007;30: 373-382.

25. Greely HT. Breaking the stalemate: a prospective regulatory framework for unforseen research uses of human tissue samples and health information. Wake Forest Law Rev 1999;34:737-766.

26. Wolf SM, Crock BN, Van Ness B, et al. Managing incidental findings and research results in genomic research involving biobanks and archived datasets. Genet Med 2012, 14:361-384

27. HUGO Ethical, Legal, and Social Issues Committee, 1995. Statement on the principled conduct of genetics research. http://www.hugo-international. org/img/statment\%20on\%20the \%20principled\%20conduct \%20of\%20 genetics\%20research.pdf. Accessed 4 April 2011.

28. Zawati MH, Van Ness B, Knoppers BM. Incidental findings in genomic research: a review of international norms. GenEdit 2011;9:1-8.

29. Cho MK. Understanding incidental findings in the context of genetics and genomics. J Law Med Ethics 2008;36:280-285, 212.

30. Beskow LM, Burke W. Offering individual genetic research results: context matters. Sci Trans/ Med 2010;2:38cm20.

31. Pulley J, Clayton E, Bernard GR, Roden DM, Masys DR. Principles of human subjects protections applied in an opt-out, de-identified biobank. Clin Trans/ Sci 2010:3:42-48.

32. Chalmers D. Genetic research and biobanks. In: Dillner J (ed). Methods in biobanking. Humana Press: New York, 2011:1-37.

33. Dressler LG. Biobanking and disclosure of research results: addressing the tension between professional boundaries and moral intuition. In: Solbakk $J \mathrm{H}$, Holm S, Hofmann B (eds). The Ethics of Research Biobanking. Springer: New York, 2009:85-99.

34. Illes J, Kirschen MP, Edwards E, et al. Ethics. Incidental findings in brain imaging research. Science 2006;311:783-784.

35. $\mathrm{NCl}$, Office of Biorepositories and Biospecimen Research, 2010. NCl best practices for biospecimen resources, revised draft document for public review and comment. http://biospecimens.cancer.gov/global/pdfs/ Revised\%20NCl\%20Best\%20Practices public\%20comment.pdf.

36. Wallace SE, Kent A. Population biobanks and returning individual research results: mission impossible or new directions? Hum Genet 2011;130: 393-401 